\title{
FEDERALISMO E FINANÇAS MUNICIPAIS NO BRASIL: UMA ANÁLISE ESPACIAL
}

Geraldo Antônio dos REIS ${ }^{1}$

João Francisco de $A B R E U^{2}$

\section{Resumo}

O presente artigo tem por objetivo proceder à análise espacial das receitas dos Municípios, bem como das relações intergovernamentais, com o propósito de avaliar as grandes assimetrias que definem o federalismo brasileiro. Para tanto, foram utilizados os meios proporcionados pela Geografia Quantitativa, especialmente nas suas interfaces com outras ciências, como a Economia. quando foram constatadas ocorrências de padrões de associação espacial para dados utilizados (2000-2015), através do Índice Global de Moran I e e do Índice Getis-Ord. Brasil.

Palavras-chave: Análise Espacial. Federalismo Fiscal. Finanças Municipais. ESDA.

\section{Abstract \\ Federalism and Municipal Finances in Brazil: a Spatial Analysis}

The purpose of this article is to analyze spatially the revenues of municipalities, as well as intergovernmental relations, in order to evaluate the great asymmetries that define Brazilian federalism. For this purpose, the means provided by Quantitative Geography were used, especially in its interfaces with other sciences, such as Economics (2000-2015), through the Moran I Global Index and the Getis-Ord Index.

Key words: Spatial Analysis. Fiscal Federalism. Municipal Finance. ESDA. Brazil.

\footnotetext{
1 Doutor - Diretor do Centro de Educação Tecnológica-Universidade Estadual de Montes ClarosUNIMONTES. Endereço: Avenida Dr. Ruy Braga, S/N - Vila Mauriceia - CEP 39401-089 Montes Claros - MG. E-mail: geraldo.reis@unimontes.br

2 Pós-Doutor e Docente Permanente do Programa de Pós-graduação em Geografia - Tratamento em Informação Espacial da Pontifícia Universidade Católica de Minas Gerais - PUC Minas. Doutor em Geografia - The University of Michigan - EUA. Endereço: Avenida Itaú, n 505 Prédio Emaús - Dom Cabral - CEP 30535-002. Belo Horizonte, MG. E-mail: jofabreu@hotmail.com
} 


\section{INTRODUÇÃO}

O artigo, ao mesmo tempo, situa-se na área de fronteira entre a Geografia das Finanças Públicas e a Economia das Finanças Públicas, e aborda as seguintes questões: em primeiro lugar, o federalismo nas suas dimensões política, geográfica e fiscal. Em segundo, a evolução do federalismo fiscal no Brasil. Em terceiro, as finanças dos Municípios brasileiros, em duas etapas distintas: a primeira, a análise e espacialização dos dados sobre receitas e transferências intergovernamentais para os Municípios brasileiros, entre os anos de 2000 e 2015. A segunda etapa, da realização da ESDA, quando é verificada a ocorrência de padrões de associação espacial para os referidos dados, através do Índice Global de Moran I (para constatar a existência de padrão do tipo cluster, aleatório ou disperso), do Índice Local de Moran I (para detectar a presença de clusters e outliers) e do Índice Getis-Ord Gi* (para identificar os hot spots e cold spots - ou áreas de maior ou menor incidência).

\section{Federalismo e Geografia}

Burgess (2012) sublinha que a relação conceitual entre o federalismo, federação, regionalismo e territorialidade constitui uma parte bem estabelecida dos estudos federais contemporâneos. Isso decorre do enraizamento da interação entre espaço, lugar e política. Mesmo que existam também elementos importantes da "nãoterritorialidade" que precisam ser observados nessa relação complexa, permanece a evidência de que Estados são, principalmente, territoriais e os cidadãos do Estado têm, entre outras coisas, uma identidade territorial distinta.

Short (2003, p. 192) argumenta que, no âmbito da relação espacial entre as pessoas e Estado, emerge a Geografia da Despesa Pública (Geography of Spending), à medida que "o gasto do dinheiro público tem implicações espaciais definidas". Mesmo nos países ricos, é possível identificar uma concentração geográfica dos gastos conforme a localização dos eleitores. Os gastos do governo são igualmente sensíveis à geografia do apoio político.

As finanças públicas emergiram como tema da Geografia Humana, em razão das implicações da distribuição espacial das receitas e despesas do governo. TrabaIhos pioneiros, como o de Bennett (1980), analisaram tanto os padrões espaciais quanto as incompatibilidade entre as geografias da geração de receita e das despesas do setor público, estabelecendo os questionamentos: quem recebe o que, onde, e a que custo? Musson (2009) ressalta que a geografia da tributação e dos gastos é a mesma em qualquer democracia. As áreas ricas proporcionam maior receita tributária per capita, mas têm menos necessidades de gasto público per capita, enquanto as áreas pobres enfrentam outra realidade realidade.

\section{A Teoria do Federalismo - Alguns Aspectos}

Bowman e Kearney (2011) argumentam que, no âmbito de uma nação, as competências e responsabilidades podem ser divididas entre os diferentes níveis de governo de três formas: através de um governo unitário, uma confederação, ou um sistema federal. Mais de $90 \%$ dos países possuem um sistema unitário, no qual a maioria, se não todo o poder legal, repousa no governo central, que pode criar ou suprimir os governos regionais ou locais, conforme a sua conveniência. 
Watts (1996) destaca as seguintes características estruturais, geralmente comuns, assumidas pelas federações como uma forma específica de sistema político federal:

a) dois níveis de governo agindo diretamente sobre os seus cidadãos;

b) uma distribuição constitucional formal da autoridade legislativa e executiva e a alocação de recursos de receita entre esses dois níveis de governo garantindo algumas áreas de autonomia genuína para cada nível;

c) existência de disposição para a representação dos entes subnacionais dentro das instituições de tomada de decisão federal, especialmente na câmara federal e senado;

d) uma Constituição suprema escrita não modificável unilateralmente e que exige o consentimento de uma parte significativa das unidades constituintes;

e) um árbitro (na forma de tribunais superiores ou provisão para referendos) para se a) pronunciar sobre os litígios entre governos;

f) processos e instituições para facilitar a colaboração intergovernamental para aquelas áreas onde as responsabilidades governamentais são compartilhadas ou inevitavelmente se sobrepõem.

Portanto, o federalismo pressupõe a dispersão geográfica do poder, com a divisão de competências e de responsabilidades entre os níveis territoriais de governo (nacional, regional e local).

\section{FEDERALISMO FISCAL, TRANSFERÊNCIAS E FINANÇAS MUNICIPAIS NO BRASIL}

O federalismo implementado no Brasil se inspirou no modelo dos Estados Unidos. Entretanto, diferentemente dos Estados Unidos, onde o federalismo foi uma técnica empregada para unir os estados e províncias que eram entidades políticas autônomas, no Brasil o federalismo serviu ao propósito de dividir aquilo que tinha sido sempre um sistema unitário de governo, e como reação ao modelo político vigente no Império, fortemente centralizado, num país de dimensões continentais, grande diversidade de regiões e distintas tradições ${ }^{3}$.

Conforme Serra e Afonso (2007, p. 31), "desde a sua criação, o sistema federal brasileiro tem passado por ciclos de contração e expansão acentuada do governo central". Rezende e Afonso (2006) denominaram tal fenômeno de "movimento pendular" da Federação brasileira. Na República Velha (1891-1930) ocorreu elevado grau de descentralização, coexistindo um governo federal débil com estados independentes fortes. No primeiro governo Vargas (1930-1945), ocorreu uma crescente concentração dos poderes no âmbito federal, cujo grande propósito era expandir e integrar o mercado interno e criar as bases para a industrialização do país. A redemocratização do país (1946-1964) favoreceu a descentralização política e fiscal, em meio à crescente concentração da atividade econômica no Sudeste, fenômeno que agravou as disparidades regionais. No regime militar (1964-1985) ocorre novo movimento pendular em direção à maior centralização. A reforma tributária, implementada logo após o golpe, ampliou a participação do governo federal na arrecadação, em detrimento dos estados e municípios, ao mesmo tempo que promoveu a modernização do sistema de arrecadação e de partilha, com a introdução de impostos sobre valor adicionado e a

\footnotetext{
${ }^{3}$ Conforme Rosenn (2005), apud Rubinstein (2011).
} 
mudança nos mecanismos de transferências para os governos subnacionais, por meio do Fundo de Participação dos Estados (FPE) e do Fundo de Participação dos Municípios (FPM).

Com o fim do regime militar e a redemocratização do país, ocorreu novo movimento de descentralização. A nova carta constitucional de 1988 empreendeu uma acentuada descentralização administrativa, transferindo muitas responsabilidades para estados, Distrito Federal e municípios - excepcionalmente nas áreas de saúde e educação. Entretanto, no campo tributário, a descentralização foi mais pronunciada do lado da repartição das receitas, do que da estrutura tributária, à medida que foram ampliados e aprimorados os mecanismos de transferências intergovernamentais. A Constituição Federal (CF) de 1988 também elevou os municípios à condição de Entes Federativos, passando a desfrutar de autonomia frente aos Estados e à União, que não podem determinar ou proibi-los de realizar ações, ainda que contribuam com parcela significativa das suas receitas (OECD, 2013).

A CF de 1988 melhorou os critérios de redistribuição dos recursos em favor das regiões menos desenvolvidas. O sistema de transferências define as várias modalidades de transferências intergovernamentais da União e dos Estados ${ }^{4}$. No Brasil, as transferências fiscais da União podem ser classificadas quanto: a) aos requisitos legais - obrigatórias: constitucionais ou legais e; discricionárias: voluntárias, para Organizações da Sociedade Civil, por delegação e específicas. b) à finalidade devolutivas, redistributivas, compensatórias, indenizatórias e mantenedoras. c) à aplicação dos recursos: vinculadas ou desvinculadas ${ }^{5}$.

O FPM é a principal transferência do governo federal para municípios e representa a segunda maior categoria, perdendo apenas para os repasses de ICMS feitos pelos estados e constitui-se em importante instrumento de redistribuição da renda nacional. Trata-se de transferência de uso incondicional, obrigatória, sem contrapartida e redistributiva. Neste caso, da União para os municípios. Desde a instituição das primeiras formas de transferências intergovernamentais no Brasil, em 1946, os percentuais de repasse sofreram significativos incrementos, possibilitando que, em meados de 2016, fossem destinados ao Fundo, 24,5\% da arrecadação líquida do IR e do IPI, arrecadados pela União6.

A CF de 1988 também determinou que estados (inclusive Distrito Federal) e municípios devem aplicar pelo menos $25 \%$ da receita de impostos (abrangendo aquela proveniente de transferências de impostos) na manutenção e desenvolvimento do ensino. Em 1996, foi instituído o FUNDEF - para o financiamento do ensino fundamental - que foi substituído, em 2006, pelo FUNDEB - ampliado para o financiamento da educação básica?.

\footnotetext{
${ }^{4}$ As informações utilizadas aqui sobre a classificação das transferências se sustentaram no documento $O$ que você precisa saber sobre as transferências fiscais da União - princípios básicos, produzido em 2015 pela STN (BRASIL, 2015b).

${ }^{5}$ Shah (2007) e Muwonge e Ebel (2015) - considerando a experiência internacional - assinalam que as transferências são classificadas em duas grandes categorias: as transferências de uso geral (também chamadas de "incondicionais") e para fins específicos (igualmente chamadas de "condicionais" ou "vinculadas"). A fonte das transferências, ou "fundo para transferências" (transfer pool), pode ser o orçamento geral do governo concedente (central ou estadual) e deve incluir uma proporção de impostos específicos.

${ }^{6}$ Da arrecadação bruta, são deduzidos restituições e incentivos fiscais. Ou seja, arrecadação líquida = arrecadação bruta - deduções.

7 A base de cálculo do FUNDEB inclui os repasses do FPE, do FPM (excluído o FPM 1\%), da LC no 87/96 (Lei Kandir), do ICMS estadual, do IPI-Exportação, do Imposto sobre Transmissão Causa Mortis e Doações (ITCMD), do Imposto sobre Propriedade de Veículos Automotores (IPVA) e do ITR devido aos Municípios. Estão inclusas ainda as receitas da dívida ativa tributária relativa aos impostos previstos, bem como juros e multas eventualmente incidentes.
} 


\section{Transferências Estaduais para os Municípios}

As duas transferências provenientes dos estados para os municípios são a cota-parte do IPVA e a cota-parte do ICMS. Os critérios de repasses estão previstos na CF e em dispositivos infraconstitucionais. Os repasses do IPVA correspondem a $50 \%$ do produto da arrecadação realizada pelo Estado, e se destinam ao município no qual o veículo for emplacado. É um exemplo de imposto sobre propriedade cuja centralização da arrecadação nas mãos do governo estadual permite considerável aumento da eficiência, em razão dos ganhos de escala, eliminação da possibilidade de guerra fiscal entre municípios na fixação da alíquota e, especialmente, redução da sonegação que geralmente acompanha a municipalização de imposto sobre propriedade - como é verificado com o IPTU. Em 2014, foram arrecadados $\mathrm{R} \$ 32,4$ bilhões ( $0,59 \%$ do PIB) ou $1,76 \%$ de toda a arrecadação de tributos no Brasil, sendo destinados para os municípios cerca de $\mathrm{R} \$ 16,2$ bilhões ou $0,295 \%$ do PIB. Mesmo que a quantidade e o valor dos veículos automotores sejam muito inferiores aos dos imóveis urbanos, a arrecadação de IPVA historicamente supera a do IPTU.

O ICMS representa uma expressiva fonte de arrecadação de impostos para os governos estaduais e a cota-parte distribuída aos municípios tem um peso importante nas suas receitas correntes, especialmente daqueles mais ricos. O critério de distribuição segue regramento constitucional, que define, no Art. 158, o repasse de $25 \%$ do produto da arrecadação para os municípios, sendo $75 \%$ transferidos de acordo com o Valor Adicionado Fiscal (VAF), apurado em cada um dos municípios (a parcela impositiva), à medida que o Estado tem de usar unicamente tal critério na partilha. A parcela restante de $25 \%$ deve ser destinada conforme previsto em legislação estadual (a parcela autônoma).

Desde a promulgação da CF, em 1988, os governos estaduais têm buscado aprimorar os mecanismos de distribuição da parcela autônoma, provocando, por essa razão, o surgimento de grande variedade de critérios para tal distribuição. No Estado de Minas Gerais, cumprindo o dispositivo constitucional, em 1991, foi aprovado o Decreto-Lei no 32.771 definindo que, além do VAF, a distribuição da cota-parte do ICMS contemplaria os municípios mineradores e ofereceria uma compensação financeira por emancipação de distritos. O mecanismo adotado favorecia os municípios que apresentavam maior dinamismo econômico.

Com o propósito de promover uma distribuição do ICMS que contemplasse mais favoravelmente os municípios com menor dinamismo econômico, e, por conseguinte, mais pobres, foi promulgada a Lei Estadual no 12.040/95, conhecida como Lei Robin Hood. Após 1995, algumas alterações nos percentuais de distribuição foram introduzidas. Contudo, somente em 2009, com a Lei Estadual n 18.030, houve uma mudança consistente, com a diminuição do peso econômico em favor de critérios sociais, configurando o "ICMS Solidário", após grande debate promovido pela Assembleia Legislativa, além da inclusão de 6 novos critérios ${ }^{8}$.

Como apresentado, as transferências no Brasil foram progressivamente cumprindo requisitos recomendados pela teoria normativa das finanças públicas, bem como pela prática de outros países, sobretudo por visar a equalização, atenuando os desequilíbrios fiscais. As transferências do FPM privilegiam o critério redistributivo e acabam desconsiderando a capacidade fiscal dos beneficiados, causando grande dependência, à medida que a maioria desses municípios ignora a necessidade de realizar esforço próprio de arrecadação. Ademais, são deficientes os critérios de

\footnotetext{
8 Sobre os critérios utilizados pela Lei do ICMS Solidário para o repasse da parcela (impositiva e autônoma) do ICMS para os Municípios, vide Reis (2016).
} 
redistribuição regional (entre regiões e entre municípios) pois a fórmula utilizada apresenta um viés acentuadamente favorável aos municípios menos populosos, com aumento expressivo das transferências per capita, comprometendo a capacidade do FPM de destinar recursos para aqueles mais pobres. As transferências vinculadas ao ICMS, por sua vez, priorizam a devolução, já que a maior parte do valor repassado aos municípios está associada ao VAF, beneficiando mais os lugares onde ocorre a receita. Essas duas modalidades de transferências representam importante fonte de recursos os municípios, mas há uma discricionariedade relativamente baixa ${ }^{9}$.

\section{Os Impostos Municipais}

Ao outorgar aos municípios a condição de ente federado autônomo, a CF de 1988 ampliou e fortaleceu a sua competência para cobrar tributos, bem como estabeleceu o total controle sobre as receitas provenientes desses tributos. Por essa razão, nas últimas décadas, a arrecadação municipal (em proporção do PIB) apresentou contínuo crescimento, passando de 0,55\%, em 1970, para 0,95\% em 1990, 1,36\%, em 2000 e 2,07\%, em 2014. Do total arrecadado pelos três níveis de governo, a arrecadação municipal saltou de 2,1\% em 1970, para 3,1\% em 1990, 4,5\% em 2000 e 6,2\% em 2014 (AFONSO; VILLELA, 1994; COSSIO, 1998; BRASIL, 2015a).

Para efetivar integralmente a sua competência tributária e ter controle total do produto da arrecadação, cada município deve regulamentar por lei, instituindo Código Tributário próprio, a cobrança dos referidos tributos em seu território, de modo coerente com o que prevê a Constituição e o CTN (AFONSO; ARAUJO; NÓBREGA, 2013).

Os dois impostos com maior potencial de arrecadação municipal são o ISS, que incide sobre a prestação de serviços a terceiros, por empresa ou profissional autônomo, com ou sem estabelecimento fixo; e o IPTU, imposto sobre a propriedade imobiliária, que é uma importante fonte de receita para os municípios e um poderoso instrumento de política urbana, à medida que pode ser ainda utilizado para conter a especulação imobiliária e promover o uso racional do solo urbano.

Não obstante a elevada sonegação, em 2014, os municípios brasileiros arrecadaram $\mathrm{R} \$ 55,3$ bilhões com o ISS (cerca de $1 \%$ do PIB), representando $3 \%$ da receita tributária dos três níveis de governo. O IPTU, pos sua vez, apesar do amparo legal, é inexplorado tanto como fonte de recursos, como instrumento de política urbana. Trata-se de imposto que apresenta um dos maiores níveis de sonegação ou isenções e sub-avaliações de valor venal (base tributária) dos imóveis, sendo a sua arrecadação praticamente inexistente em muitos municípios do país. Em 1970, a arrecadação de IPTU representava 0,32\% do PIB, elevando-se para 0,44\% do PIB em 1991 e alcançou 0,51\% do PIB em 2014 (AFONSO; VILLELA, 1994; COSSIO, 1998; BRASIL, 2015a).

Em 2014, o ISS representou 48,31\% da arrecadação municipal (1,00\% do PIB), o IPTU, 24,63\% (0,51\% do PIB), o ITBI, 9,7\% (0,20\% do PIB), as Contribuições para o Regime Próprio Previdência Municipal, $7,25 \%(0,15 \%$ do PIB) e os Outros Tributos Municipais, $10,14 \%(0,21 \%$ do PIB). Isto é, ISS, IPTU e ITBI responderam por $82,64 \%$ das receitas municipais, mostrando que as outras fontes, como taxas e contribuições de melhoria, possuem papel marginal (BRASIL, 2015a).

\footnotetext{
9 Sobre as transferências no Brasil, vide Piancastelli, Boueri e Lima (2006), Mendes, Miranda e Cosio (2008) e Ter-Minassian (2012). Piancastelli, Boueri e Lima (2006) salientam que o sistema de transferência são essencialmente redistributivas, enquanto Ter-Minassian (2012) afirma que o sistema prioriza a devolução como critério de repasse, privilegiando assim os lugares onde ocorre a receita.
} 
A participação do imposto sobre propriedade imobiliária no Brasil - tendo o IPTU um papel preponderante - é bem menor que o registrado em outros países. Por exemplo, em 2009 alcançou cerca de 3\% do PIB nos Estados Unidos, enquanto representava 0,39\% do PIB no Brasil. (REIS, 2016) ${ }^{10}$.

\section{Métodos e Técnicas}

\section{Exploratory Spatial Data Analysis (ESDA): a Dependência Espacial}

Para Oyana e Margai (2016) a noção de dependência espacial capturada pela Primeira Lei de Tobler permite destacar dois aspectos-chave da dependência espacial: 1) dependência variável espacial (autocorrelação espacial e correlação espacial) e 2) a dependência das relações espaciais (homogeneidade espacial e heterogeneidade espacial). Tais estruturas espaciais subjacentes têm grandes implicações na forma como são formulados os problemas de pesquisa, a amostragem de dados, a medição e como hipóteses são testadas.

Com os avanços ocorridos nos últimos 50 anos na teoria, na técnica e nos recursos computacionais - especialmente por meio dos SIG's - foram proporcionados novos horizontes para a análise espacial, com o aprimoramento da ESDA ${ }^{11}$. Anselin (1999) definiu a ESDA como

[...] o conjunto de técnicas para descrever e visualizar distribuições espaciais, identificar locais atípicos (outliers espaciais), descobrir padrões de associação espacial (clusters espaciais), e sugerir diferentes regimes espaciais ou outras formas de instabilidade espacial ou não estacionariedade espacial. (ANSELIN, 1999, p 121).

A ESDA abre perspectivas inéditas para a identificação de padrões de comportamento das variáveis geográficas, com a combinação de técnicas provenientes da Estatística Espacial e da Econometria Espacial.

Dentre os recursos propiciados pela ESDA estão: a produção de indicadores de correlação e dependência espacial (clusters, outliers, hot spots); a visualização da distribuição espacial, por meio de técnicas de visualização cartográfica, se configurando ainda como uma etapa descritiva inicial e indispensável da modelagem econométrica espacial, que antecede tanto a análise confirmatória (estimação e contrastes); bem como o próprio exercício de predição espacial.

Os termos autocorrelação espacial e dependência espacial podem ser usados como sinônimos. A dependência espacial indica que o valor de uma variável de interesse numa certa região $i$ depende do valor dessa variável nas regiões vizinhas $j$ (ANSELIN, 2003; ALMEIDA, 2004, 2012). Esse conceito pode ser expresso como:

$$
\begin{aligned}
& y_{i}=f\left(y_{j}\right) \quad i=1, \ldots, n \text { e } i \neq j \\
& y_{i \leftrightarrow y_{j}}
\end{aligned}
$$

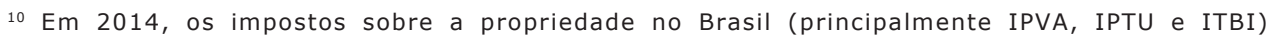
representam somente $1,40 \%$ do PIB (4,17\% do total) (BRASIL, 2015a). Em outros países, tal participação é muito mais significativa: 2,60\% no Japão (2008), 3,08\% nos Estados Unidos (2009), 3,60\% no Canadá (2009), 4,38\% no Reino Unido (2009) e 4,48\% na França (2009) - de acordo com dados do Government Finance Statistics (GFS)/FMI.

11 Bivand (2010) afirma que a ESDA utilizada na Estatística Espacial, Econometria e Geoestatística Espaciais, foi desenvolvida a partir da exploratory data analysis (EDA).
} 
O esquema mostra que há interação entre a variável de interesse $y$ da unidade espacial $i$ com a mesma variável localizada na unidade espacial contígua a ela, denominada $j$. Ou seja, inexiste independência entre as observações do conjunto de dados. Além disso, a dependência no espaço é bidirecional, ao contrário do que ocorre com as séries de tempo cuja direção da interação e a dependência no tempo ocorrem unidirecionalmente, do passado para o presente e do presente para o futuro.

A autocorrelação espacial permite assinalar a concentração ou dispersão dos valores de uma variável no espaço. As distribuições das observações podem ser: 1) aleatória: qualquer ponto tem a mesma probabilidade de ocorrer em qualquer local, e a posição de qualquer ponto não é afetada pela posição de qualquer outro ponto. Consequentemente, inexiste uma causa que explique a distribuição; 2) uniforme ou não aleatória: cada ponto está tão longe de todos os seus vizinhos quanto possível; e 3) cluster (agrupada/aglomerada): muitos pontos estão aglomerados e há grandes áreas onde poucos estão localizados. Quando agrupamentos estatisticamente significativos são identificados, são oferecidas pistas importantes sobre os motivos que levaram à formação de tais agrupamentos.

Outro efeito importante que está associado à heterogeneidade espacial: de acordo com Sankey (2008), um processo espacial é chamado estacionário ou homogêneo se as propriedades estatísticas dos valores dos atributos são independentes da localização. Em contraste, um processo espacial é não estacionário ou heterogêneo se a média, variância e covariância dos valores de atributos observados mudam através de uma região de estudo.

As estatísticas de autocorrelação global permitem testar a hipótese de que existe uma associação significativa de valores entre as regiões vizinhas, contra a hipótese nula de que a variável é distribuída de forma completamente aleatória no espaço. Dentre as estatísticas globais mais utilizadas estão Índice Global de Moran I e $G(d)$, de Getis e Ord. Contudo, nas situações em que predomina uma instabilidade significativa na distribuição espacial da variável sob estudo (processos não estacionários espacialmente), as estatísticas globais são insuficientes. Por essa razão, é necessário o emprego de forma complementar das chamadas estatísticas locais, como análise de clusters e outliers (Índice Local de Moran I) e Análise de Hot Spots (Índice Getis-Ord $G_{i}^{*}$ ). Para Fotheringham, Brunsdon e Charlton (2002), a estatística local permite que as diferentes tendências na distribuição de uma variável possam existir no espaço. Em algumas partes da área de estudo, por exemplo, valores elevados podem ser agrupados; em outras partes, pode haver uma mistura de valores altos e baixos. Estas diferenças não seriam aparentes para o cálculo de uma estatística global.

A abordagem metodológica do presente trabalho contempla dois momentos: o primeiro, a análise e espacialização dos dados sobre receitas, despesas e transferências intergovernamentais para os Municípios brasileiros, entre os anos de 2000 e 2015. O segundo momento, da realização da ESDA, quando é verificada a ocorrência de padrões de associação espacial para os referidos dados, através do Índice Global de Moran I (para constatar a existência de padrão do tipo cluster, aleatório ou disperso), do Índice Local de Moran I (para detectar a presença de clusters e outliers) e do Índice Getis-Ord Gi* (para identificar os hot spots e cold spots - ou áreas de maior ou menor incidência) ${ }^{12}$. A espacialização dos dados e realização da ESDA é feita com o uso do Arcgis.

A elaboração desses índices conta com as seguintes bases de dados: a) da STN/Ministério da Fazenda, para as informações sobre as finanças municipais; b) do

\footnotetext{
12 Sobre tais indicadores, vide Rogerson (2001), Lloyd (2010), Wang (2015) e Oyana e Margai (2016). Vide ainda <http://help.arcgis.com>.
} 
PNUD/Fundação João Pinheiro/IPEA/IBGE, para os indicadores sociais, como renda familiar, proporção de pobres e IDHM; e c) do PIB municipal e malhas digitais, do IBGE.

A espacialização dos resultados por meio de SIG tem por intuito estimar a autocorrelação espacial e identificar clusters e outliers espaciais entre os municípios. Para tanto, é realizada a análise exploratória (ESDA), que permite descrever a distribuição espacial, assinalar a existência de padrões de associação espacial (clusters espaciais), bem como a ocorrência e as formas de instabilidade espacial (ALMEIDA, 2004, 2012). Isto é, a análise espacial possibilita a compreensão dos efeitos decorrentes da dependência espacial e da heterogeneidade espacial ${ }^{13}$.

O primeiro passo envolve o cálculo do Índice Global de Moran I, que corresponde a uma extensão espacial do Índice de Correlação linear de Pearson ${ }^{14}$. No Arcgis a ferramenta Spatial Autocorrelation (Moran I) calcula o valor do escore $z$ e o valor $p$ que indicam se se deve rejeitar a hipótese nula - a completa aleatoriedade espacial (CSR), seja das entidades ou dos valores associados a essas entidades. Quando o valor de $p$ é pequeno e o valor absoluto do escore $z$ é grande o suficiente para que ele fique fora do nível de significância estabelecido, a hipótese nula deve ser rejeitada. Se o valor do índice é maior que 0 , o conjunto de dados exibe um padrão de cluster. Se o valor for menor que 0 , o conjunto de dados exibe um padrão disperso.

O segundo passo envolve o cálculo do Índice Local de Moran $I$, com o intuito de detectar a existência de clusters e outliers espaciais. A estatística de autocorrelação espacial local fornece uma medida, para cada unidade na região, da tendência da unidade em ter um valor de atributo que está correlacionado com valores verificados em áreas próximas. Para tanto, é utilizada a ferramenta do Arcgis que calcula o Índice Local de Moran $I$, o escore $z$, o valor $p$, e um código correspondente ao tipo de cluster/outlier para cada característica (COType). O escore $z$ e o valor $p$ indicam se a aparente similaridade (um cluster espacial de valores similares) ou a falta de similaridade (um outlier espacial) é mais pronunciada do que o que se espera numa distribuição aleatória ${ }^{15}$. Portanto, o objetivo é localizar agrupamentos de valores similares, além de avaliar se uma observação é muito singular em relação à vizinhança, usando os seguintes critérios (COType):

1) $\mathrm{HH}$ (ALTO/ALTO): cluster de valores altos (unidades com valores altos localizam-se próximas às unidades com valores altos);

2) LL (BAIXO/BAIXO): cluster de valores baixos (unidades com valores baixos localizam-se próximas às unidades com valores baixos);

3) HL (ALTO/BAIXO): outlier com valor alto em relação a vizinhos de baixo valor (unidades com valores altos próximas às unidades com valores baixos);

4) $\mathrm{LH}$ (BAIXO/ALTO): outlier com valor baixo em relação a vizinhos de alto valor (unidades com valores baixos próximas às unidades com valores altos).

O terceiro passo envolve o cálculo do Índice Getis-Ord $G^{*}{ }^{*}$. O Índice permite identificar hot spots, determinando assim se existe algum clustering estatisticamente

\footnotetext{
13 Sobre a metodologia de análise espacial vide Almeida (2004, 2012), Lesage e Pace (2009), Leung (2010), Pinheiro (2010) e Souza, Silva e Martins (2011).

14 O índice é análogo ao coeficiente de correlação convencional, cujos valores de $I$ também variam de -1 a +1 , quantificando o grau de autocorrelação espacial existente, de tal modo que: a) -1 autocorrelação espacial negativa ou inversa; b) 0 significa aleatoriedade; e c) +1 significa autocorrelação espacial positiva ou direta.

15 Conforme informações contidas em: http://help.arcgis.com/es/arcgisdesktop/10.0/help/ index.html\#/na/005p0000000z000000/
} 
significativo no padrão espacial dos dados, ao mesmo tempo em que permite localizar as regiões onde determinado fenômeno ocorre com maior incidência. A Análise de Hot Spot do Arcgis calcula o Índice Getis-Ord $G^{*}{ }_{i}$ para cada característica de um conjunto de dados. O escore $z$ resultante mostra onde os locais com valores altos ou baixos se agrupam espacialmente. Para ser um hot spot estatisticamente significativo, uma característica precisa ter um valor alto e estar cercada por outras com valores igualmente altos. A soma local para uma característica e os seus vizinhos é comparada proporcionalmente à soma de todas as características; quando a soma local é muito diferente da soma local esperada, e a diferença é muito grande para ser um resultado aleatório, como consequência, se obtém um escore $z$ estatisticamente significativo.

Uma etapa complementar envolve a definição da matriz de ponderação espacial $(W)$ representa uma etapa delicada e imprescindível da ESDA $^{16}$. A finalidade da matriz é expressar um determinado arranjo espacial das interações entre as regiões que estão sendo objeto de estudo. A hipótese que norteia a confecção de tais arranjos é que as regiões mais próximas possuem maior interação que as regiões mais distantes.

Uma matriz de vizinhança pode ser elaborada de diversas maneiras, sendo delineadas por contiguidade, proximidade a partir de alguma regra de potência ou ainda por algoritmo específico, para a determinação de um número ideal de vizinhos (GETIS; ALDSTADT, 2010). Ou seja, a proximidade entre as regiões pode envolver critério não somente geográfico, mas também socioeconômico ou ainda outro mais adequado ao fenômeno estudado, como critérios culturais e pesos institucionais (ALMEIDA, 2012).

Para o caso da malha municipal brasileira, constituída por feições irregulares, assimétricas e de tamanhos muito variados, a utilização de matrizes binárias de contiguidade de primeira ordem ou a utilização de critérios que valorizem os vizinhos imediatos podem distorcer as estimativas dos municípios. A utilização de um raio de distância fixa, nesse caso, contribui para a suavização da média e da variância local, bem como estabelece um limiar independentemente do número de vizinhos, reduzindo o problema da irregularidade de feições quanto a identificação de unidades que compõem a vizinhança imediata.

Entre os diversos instrumentos de análise do Índice Global de Moran $I$, e do Índice Local de Moran $I$ e Índice Getis-Ord $G^{*}$, optou-se pelo uso da matriz de vizinhança zona de distância fixa, pois o interesse residiu em definir uma vizinhança mínima em que pelo menos um vizinho é selecionado ${ }^{17}$. As bandas fixas oferecem redução de flutuações locais e mostram padrões regionais. Simulações mostram que a significância estatística do Índice Global de Moran é crescente com a distância, mas o respectivo índice decai com a distância. Assim, a distância ótima seria aquela que consiga oferecer um valor mais elevado para o Índice de Moran, com a maior significância estatística e que define um mínimo de vizinhos necessários para as unidades de análise. Ou seja, que não exclua nenhum município em seu cômputo geral. Para tanto, optou-se por utilizar o mesmo raio de vizinhança definido pelo Arcgis, de 313,84 quilômetros.

\footnotetext{
16 Sobre matrizes de ponderação espacial, vide Almeida (2012).

17 Uma alternativa poderia ser a zona de indiferença. Contudo, ela é recomendada para distâncias mais curtas e para calculo de custo de decisão, o que não é o objetivo desse trabalho. A zona de indiferença é interessante para avaliar a probabilidade de deslocamento para o trabalho, uma vez que trabalhadores optam por uma área mínima de interesse de deslocamento, sendo que a possibilidade de emprego a partir dessa distância sofre um decaimento com a mesma. No estudo aqui de interesse, essa lógica não se aplica.
} 
A matriz de vizinhança zona de distância fixa apresenta uma ligeira queda no Índice Global de Moran, principalmente, em relação ao inverso da distância e ao inverso da distância ao quadrado, mas igualmente gera um incremento da significância, medido pelo escore $z$.

\section{A ARRECADAÇÃO MUNICIPAL NO FEDERALISMO BRASILEIRO}

\section{Análise dos Padrões Espaciais das Finanças dos Municípios}

A produção de indicadores de correlação e dependência espacial (clusters, outliers, hot spots), bem como a espacialização desses indicadores, com a geração de mapas temáticos, permite a compreensão das grandes disparidades na capacidade fiscal dos Municípios brasileiros.

O resultado do trabalhho permite constatar que o dinamismo econômico afeta a capacidade fiscal dos municípios. As assimestrias nos indicadores das finanças municipais refletem, em sua maior parte, os significativos desníveis econômicos entre as regiões do país, condicionados em sua maior parte, pela loalização dessas regiões. Em síntese, foi possível observar que o dinamismo econômico (PIB per capita), a qualidade de vida (IDHM), a incidência de pobreza e, por conseguinte, a capacidade fiscal dos municípios, têm forte relação com a sua localização: os municípios mais ricos e dinâmicos estão próximos de outros municípios semelhantes, formando aglomerações (clusters); enquanto aqueles mais pobres e mais dependentes de recursos formam aglomerações com outros na mesma condição.

Enquanto o baixo dinamismo econômico - e todas as suas implicações - une municípios localizados sobretudo no Norte e Vales do Jequitinhonha e Mucuri, em Minas Gerais, e os estados do Norte e do Nordeste, há uma aglomeração de municípios cujos indicadores são mais satisfatórios, especialmente no Sul e Triângulo Mineiro, Sul do Espírito Santo, Rio de Janeiro e São Paulo, e a região Sul do Brasil. Essas duas áreas são separadas por uma longa faixa composta pelos municípios cujos valores não são estatisticamente significativos. O desenho apresentando pelos mapas explicita a divisão Norte-Sul, trazendo evidências de que a segregação espacial constitue mais um componente da diversidade geográfica do país.

As estatísticas de autocorrelação global (Índice Global de Moran I) permitem testar a hipótese de que existe uma associação significativa de valores entre as regiões vizinhas, contra a hipótese nula de que a variável é distribuída de forma completamente aleatória no espaço - completa aleatoriedade espacial (CSR). Isto é, o Estudo mostra que o dinamismo econômico, a qualidade de vida, a incidência de pobreza e a capacidade fiscal não estão aleatoriamente distribuídos no espaço, sendo constatada existência de autocorrelação espacial positiva. Entretanto, em algumas partes do país, é possível observar uma mistura de valores altos e baixos, característica não detectada pelo cálculo de uma estatística global, tornando assim necessário o uso de estatísticas locais (Índice Local de Moran $I$ e Índice Getis-Ord $G^{*}{ }_{i}$ ).

Em virtude da sua grandeza territorial, o Brasil comporta imensa diversidade econômica, social, cultural e geográfica, e mesmo assim logrou êxito em preservar a sua unidade política e institucional e, certamente, o federalismo concorreu para a construção dessa unidade. A figura 1 nos fornece uma evidência das grandes disparidades econômicas, por meio das informações referentes ao PIB per capita dos municípios em 2013, indicador fundamental para se avaliar a capacidade de geração de riquezas e, consequentemente, a capacidade fiscal desses entes. É possível observar a concentração de áreas dinâmicas no Sul, Sudeste e Centro-Oeste do Brasil. 

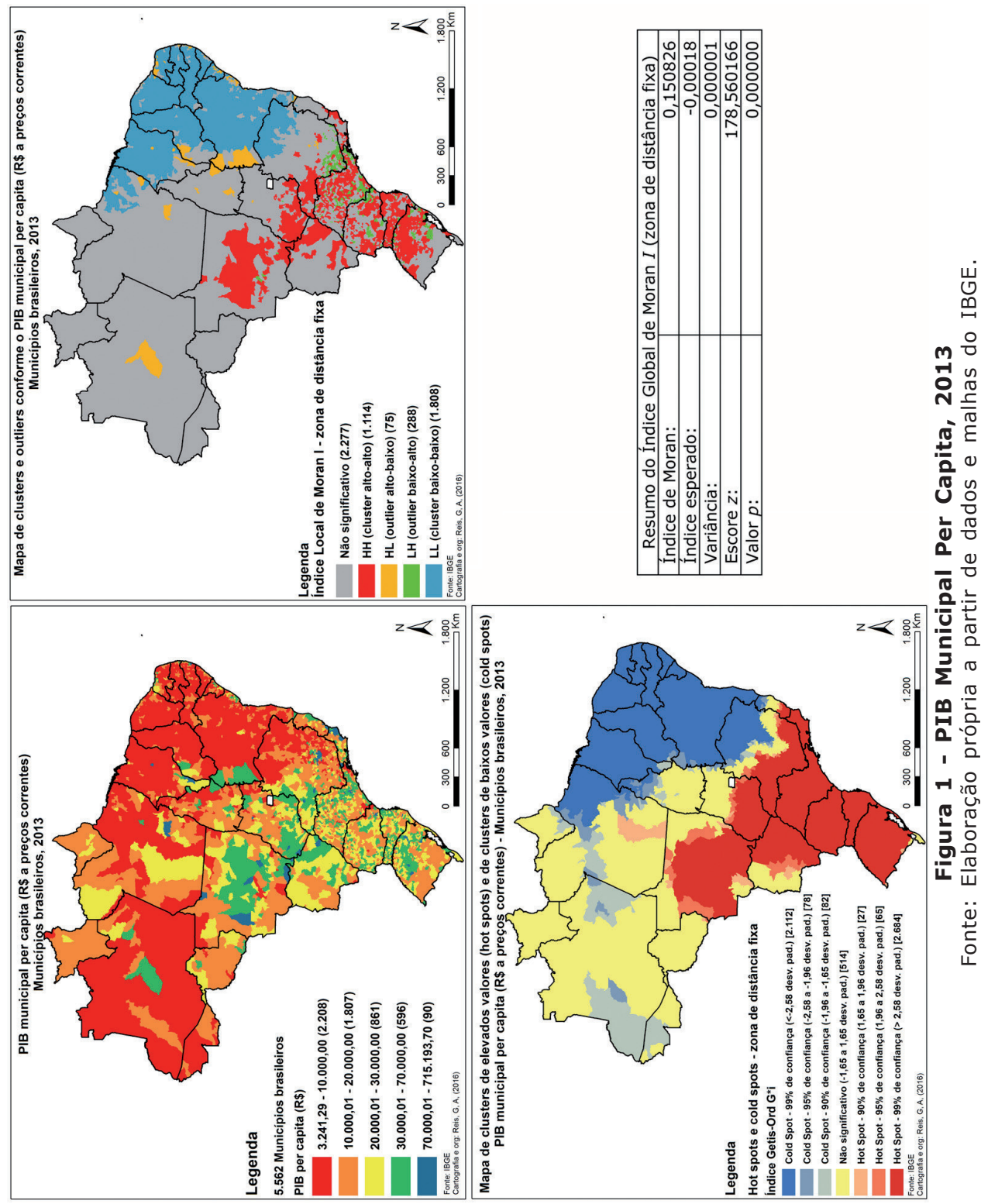
Na figura1 está exposta ainda a tabela com o resumo do Índice Global de Moran I, que permite medir o grau de autocorrelação espacial existente; o mapa de clusters e outliers, derivado do Indice Local de Moran I; e o mapa de hot spots e cold spots, a partir do Índice Getis-Ord $G_{i}{ }^{*}$. Como o valor do Índice Global de Moran $I$ é maior que $0(0,150826)$, o conjunto de dados revela um padrão de cluster. Existem clusters de elevados valores de PIB per capita (cluster alto-alto), agrupados nas regiões mais ricas e clusters com baixos valores (cluster baixo-baixo) concentrados particularmente no Norte e Vales do Jequitinhonha, em Minas Gerais, e no Nordeste do país. Há também uma tendência à aglomeração de algumas variáveis, mas também uma concentração das desigualdades locais, destacadas pela distribuição dos outliers alto-baixo ou baixo-alto. Isso indica algumas ilhas de prosperidade circundadas por municípios pobres em áreas do Nordeste, como o Extremo-Oeste da Bahia, bem como ilhas de pobreza nas regiões mais dinâmicas do país, como no Sul de Minas Gerais, no Vale do Ribeira, em São Paulo, e algumas áreas do Paraná e do Rio Grande do Sul.

$O$ Índice Getis-Ord $G_{i}^{*}$ possibilita abordagem alternativa para identificar as aglomerações, por meio de hot spots e cold spots, como se observa na figura 1. As áreas de elevados valores (hot spots) e de baixos valores (cold spots) seguem padrão semelhante ao identificado no mapa pelo Índice Local de Moran $I$, mostrando uma nítida divisão do país, em duas áreas muito bem definidas e opostas: uma área de hot spots, nos lugares cujas economias são as mais dinâmicas do país, confrontados por municípios que compõem área de cold spots, situada principalmente nas regiões mais pobres. Essas duas áreas são separadas por uma faixa formadada por municípios cujos valores não são estatisticamente significativos.

Mesmo que, por um lado, exista aleatoriedade espacial dos dados para algumas regiões do Brasil, e, por outro, os dois indicadores locais (Índice Local de Moran $I$ e Índice Getis-Ord $G_{i}^{*}$ ) sejam distintos, os resultados produzidos exprimem similaridade quanto à indicação, em mapas temáticos, de aglomerações espaciais significativas para os referidos dados. A análise dos demais indicadores, relacionados às finanças dos municípios ${ }^{18}$, revela uma forte interação com os indicadores sociais e econômicos avaliados (PIB e renda familiar per capita, proporção de pobres e IDHM), tanto do ponto de vista da distribuição espacial, quanto das intensas disparidades encontradas entre os municípios. Logo, baixos indicadores de renda familiar e de pobreza estão diretamente associados a níveis insatisfatórios de IDHM, ao mesmo tempo em que carregam a evidência de que em muitos lugares a população enfrenta grandes carências, impossíveis de serem providas pelo mecanismo do mercado, requerendo assim a intervenção ativa do poder público. Nesses lugares, o poder público padece de insuficiência crônica de recursos em razão da sua limitada capacidade fiscal, explicada principalmente pelo baixo dinamismo econômico.

$\mathrm{Na}$ figura 2, estão expostos os mapas referentes à média anual, entre 2000 e 2015, da receita orçamentária municipal por habitante, a preços de $2015^{19}$. O mapa de clusters e outliers mostra a existência de significativas aglomerações de municípi-

\footnotetext{
18 Optou-se por realizar o cálculo da média para uma série de 16 anos para cada indicador selecionado (entre 2000 e 2015) considerando que, para alguns municípios, tal média envolve número menor de anos. Naqueles casos cujos dados do município não estavam disponíveis para o ano, foi calculada uma média com quantidade menor de anos. Assim, o referido procedimento permitiu que fosse estimada uma média com número menor de anos, como uma proxy, assegurando que todos os municípios fossem contemplados com dados para cada indicador, evitando inexistência de informação. Dentre os indicadores selecionados estão: receita tributária por habitante, receitas correntes por habitante, arrecadação de IPTU e de ISS por habitante, participação do IPTU receita tributária, transferências correntes por habitante, participação das transferências correntes na receita orçamentária, participação do FPM e dos repasses de ICMS nas transferências correntes e repasses do IPVA por habitante.

19 Os valores dos foram deflacionados pelo deflator do PIB nacional, tendo 2015 como ano-base.
} 
os, conforme calculado pelo Índice Local de Moran $I$. De um lado, estão os municípios com baixos valores de receita orçamentária municipal por habitante (cluster baixobaixo) e, em condição oposta, se localizam municípios que formam clusters de elevados valores, agrupados nas regiões mais ricas.

A ocorrência de clusters de elevados e de baixos valores pode ser ainda detectada no mapa da figura 2, derivado do Índice Getis-Ord $G_{i}{ }^{*}$, em que municípios formam uma área de hot spots, que se confunde com os lugares cujas economias são as mais dinâmicas do país, confrontados por municípios que compõem área de cold spots, situada, basicamente nas regiões mais pobres.

A figura 2 também destaca o mapa temático que retrata a existência, de um lado, de clusters de valores baixos - reduzida participação da receita tributária na receita orçamentária - para muitos municípios concentrados no Nordeste, Norte e Vales do Jequitinhonha e do Mucuri de Minas Gerais. E, de outro, as aglomerações de valores elevados, formadas por municípios localizados em São Paulo, Minas Gerais (singularmente Sul e Triângulo), no interior do Rio Grande do Sul, de Santa Catarina, do Paraná, de Goiás, do Mato Grosso do Sul, e do Rio de Janeiro, além de algumas regiões metropolitanas desses Estados.

É possível igualmente atestar as desigualdades locais, pela distribuição dos outliers alto-baixo ou baixo-alto. Da mesma forma, a ocorrência de municípios formando áreas de cold spots, constrastando com outros formando áreas de hot spots.

A reduzida participação da receita tributária na receita orçamentária para um número expressivo dos municípios reflete o baixo esforço de arrecadação e a elevada dependência das transferências. Há de se ressaltar que, quanto menor o município, menor a participação das receitas próprias nas receitas orçamentárias ${ }^{20}$ (REIS, 2016). Enquanto o FPM é fundamental para os municípios das regiões mais pobres, as transferências provenientes da cota-parte do ICMS têm um peso significativo para os municípios das regiões mais dinâmicas do país.

Os municípios menos populosos são os mais contemplados proporcionalmente, com os repasses do FPM, causando assim distorções à medida que eles também se localizam nas regiões ricas do país. Por isso, são duplamente favorecidos pelos critérios de transferências, pois usufruem de valores maiores provenientes dos repasses de ICMS em razão do seu elevado dinamismo econômico, ao mesmo tempo em que são privilegiados pelos critérios de repasse do FPM. (REIS, 2016).

Entretanto, tais transferências não estão contemplando os municípios mais carentes de recursos. Ou seja, repete-se aqui outro desequilíbrio espacial, pois os lugares mais pobres não aparecem entre aqueles mais favorecidos por transferências de maior valor. Muito embora nas últimas décadas os mecanismos de transferências tenham sido desenhados e implementados buscando atenuar tais assimetrias, persiste como desafio assegurar que os municípios comprovadamente carentes - especialmente os localizados nas regiões mais pobres - sejam efetivamente melhor contemplados, como meio indispensável para a superação da deficiente capacidade por eles enfrentada de prover serviços públicos de boa qualidade. Certamente, o aprimoramento dos critérios de repasse poderia ser acompanhado de medidas destinadas a induzir ao maior esforço fiscal.

\footnotetext{
20 A participação da receita tributária nas receitas locais é reduzida no Brasil $(22,8 \%)$, se comparada com outros países federais como Canadá $(39,4 \%)$ e Estados Unidos $(48,4 \%)$. Isso evidencia tanto a significativa concentração das melhores fontes de receita nos governos estaduais e federal, quando o baixo esforço fiscal da maioria dos Municípios do país. Consequentemente, a fatia das transferências na receita local nos municípios brasileiros $(58,8 \%)$ se situa num nível bem mais elevado que o observado em países federais como Estados Unidos $(42,1 \%)$ e Canadá $(43 \%)$, evidenciando assim a grande dependência de transferências provenientes dos níveis superiores. (REIS, 2016).
} 

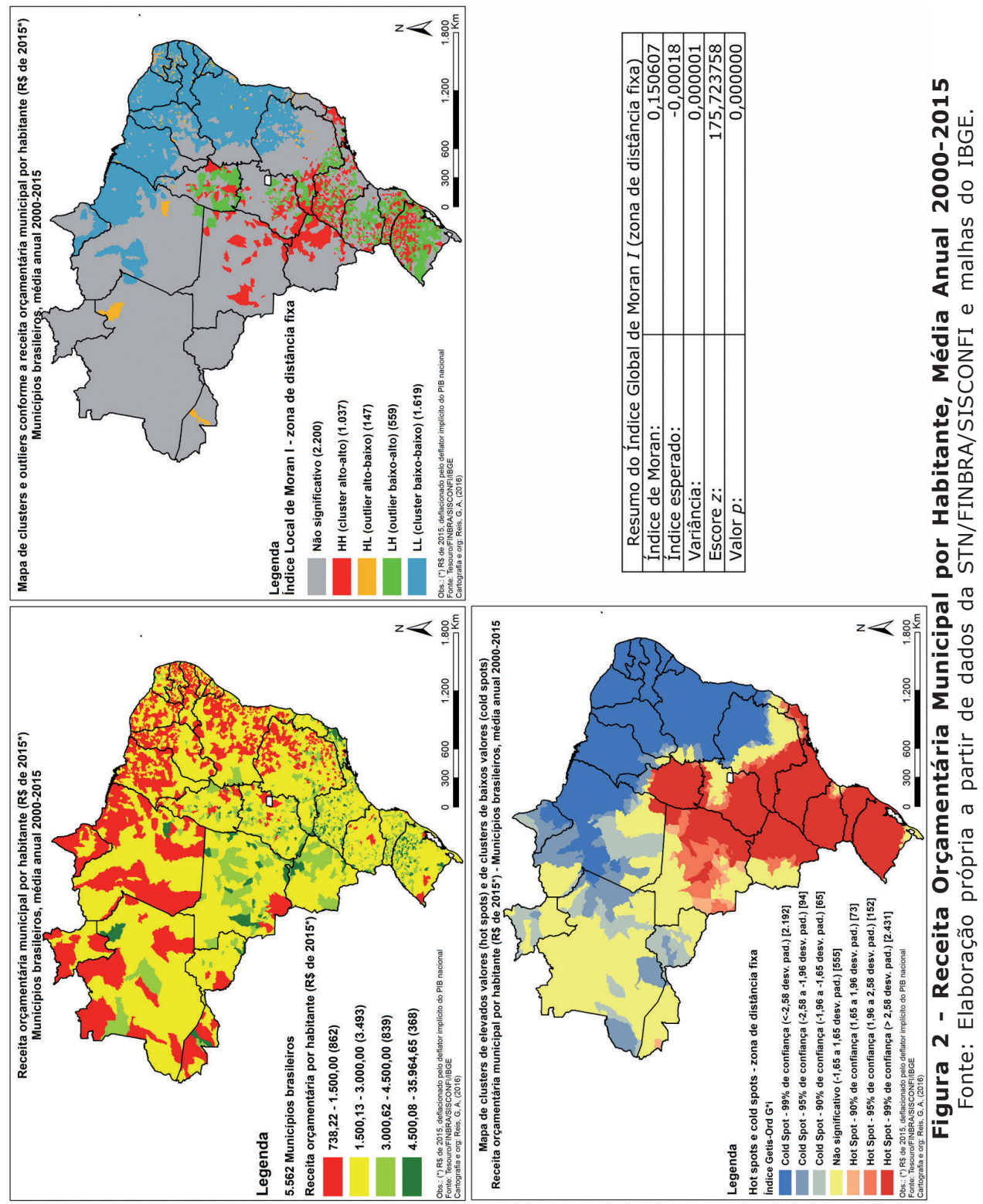

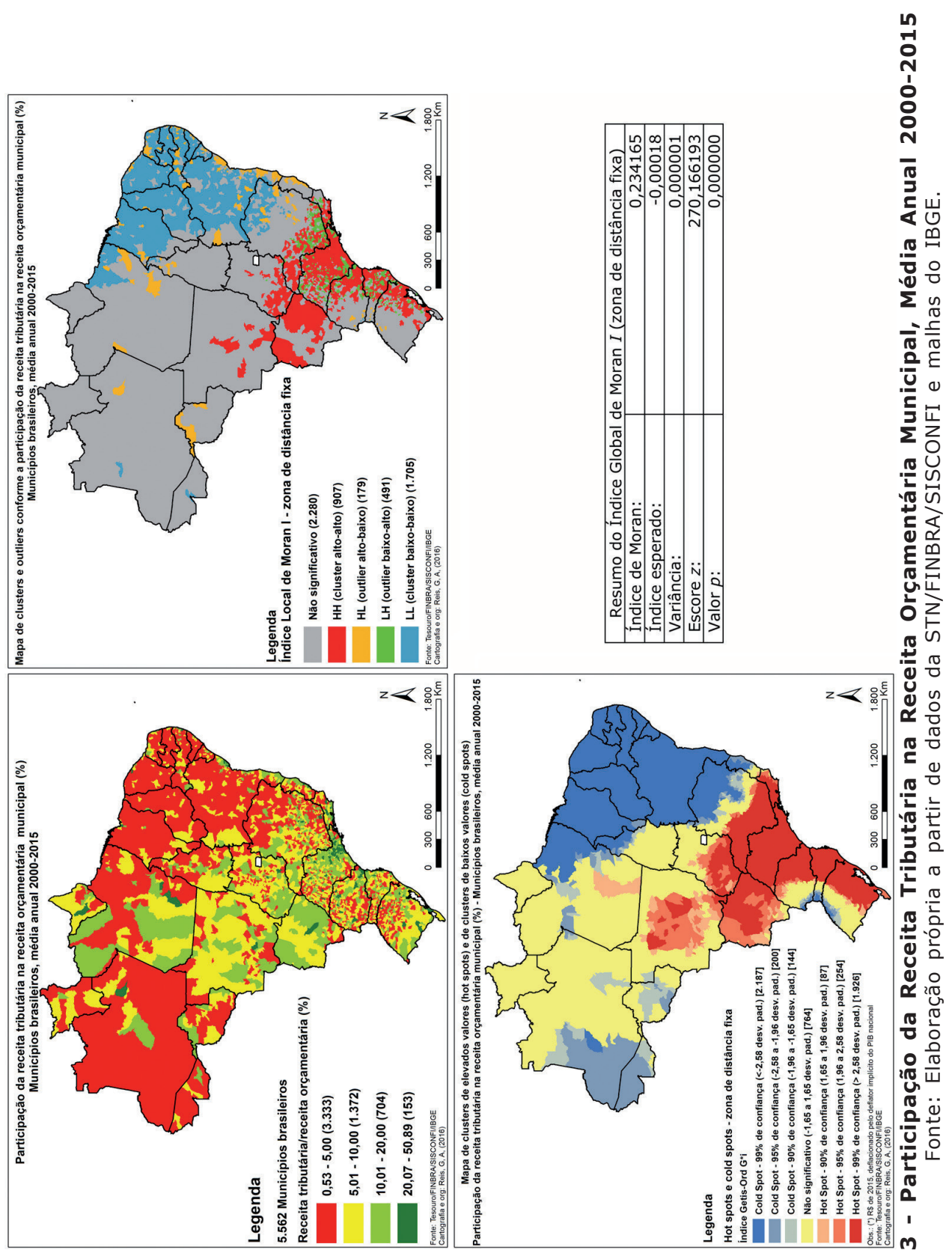
Assim, há uma visível fratura entre regiões ricas e pobres também no que diz respeito à capacidade do poder público municipal cumprir suas funções. E, processos de descentralização que não reflitam tal fratura poderão acarretar o aprofundamento das desigualdades, em virtude das habilidades muito diferenciadas entre os municípios para o cumprimento das suas responsabilidades. Conforme advertiu McLure (1998), a efetivação da descentralização está condicionada ao controle das fontes próprias de receitas pelos governos subnacionais. A autonomia fiscal ficaria seriamente comprometida se os estados e municípios permanecerem "sob o jugo financeiro do governo central" (McLURE, 1998, p. 3). A advertência do autor pode ser complementada por outra: a descentralização pode fracassar em razão dos obstáculos representados pela frágil base econômica que os governos subnacionais das regiões pobres enfrentam para conquistar maior autonomia fiscal.

A espacialização dos dados confirmou a presença de grandes disparidades na capacidade fiscal - sobretudo receitas e despesas por habitante - determinadas pela localização dos municípios. Tais disparidades são acompanhadas pelos mesmos tipos de desequilíbrios espaciais verificados no campo econômico e social. Os mapas permitem ainda identificar com clareza padrões espaciais muito bem definidos para a praticamente todos os indicadores de finanças municipais, com significativas aglomerações de municípios com valores baixos ou elevados.

As assimetrias ilustram ser inviável pensar o federalismo brasileiro a partir de pressupostos como aqueles recomendados pela Escola da Escolha Pública e pelos adeptos do federalismo competitivo.

Ainda que os mecanismos de transferências tenham sido aprimorados nas últimas décadas, com o propósito de favorecer os municípios mais carentes, a espacialização dos dados indica que as maiores concentrações de municípios com elevados valores de transferências estão nos lugares mais ricos e dinâmicos, contrariando assim tal propósito. De um lado, estão as distorções associadas aos critérios de repasse do FPM, inclinados a contemplar os municípios menos populosos - muitos deles também situados nas regiões mais ricas. De outro, o relevante papel que os repasses relacionados ao ICMS têm desempenhado para os municípios dos estados mais ricos, que são, em sua maior parte, devolutivos, seguindo a participação no VAF.

\section{CONCLUSÕES}

As diversas concepções teóricas discutidas são praticamente unânimes em caracterizar o federalismo como um sistema fundado na dispersão geográfica do poder à medida que viabiliza a divisão de competências e de responsabilidades entre os níveis territoriais de governo (nacional, regional e local).

A análise do padrão espacial das receitas e gastos constitui objeto da Geografia das Finanças Públicas. Por meio dessa análise, é possível identificar as profundas assimetrias no âmbito do federalismo brasileiro, especialmente no que diz respeito à capacidade fiscal, que se manifestam como um problema de desequilíbrio espacial.

A análise dos dados das finanças públicas dos municípios entre 2000 e 2015, oferece evidências de que os municípios brasileiros são muito dependentes das transferências, inclusive os que se situam nas regiões mais dinâmicas do país. Tal dependência reflete o acentuado hiato fiscal - entre os mais elevados do mundo - em virtude da concentração das principais fontes de receitas nos níveis superiores de governo. Essa forte dependência também reflete o baixo esforço fiscal - a baixa arrecadação de IPTU é um exemplo. No caso da maioria dos municípios localizados 
nas regiões mais pobres ou de pequeno porte populacional, o esforço fiscal é ainda menor. $\mathrm{E}$, no caso dos municípios situados nas regiões mais pobres, a frágil base econômica limita significativamente a capacidade de arrecadação - consequência dos desequilíbrios horizontais.

Os resultados apresentados no presente trabalho de pesquisa igualmente fornecem evidências de que existem significativas interações espaciais nos indicadores analisados, conforme argumentado pela Geografia Quantitativa. Da mesma forma, de que a desigualdade na capacidade fiscal tanto é consequência, quanto pode concorrer para a reprodução dos desequilíbrios espaciais na Federação brasileira.

Como comentário final, ressalta-se que a análise espacial potencializada pela Ciência Geográfica - em geral - - e pela Geografia Quantitativa - em particular oferecem grandes oportunidades para melhorar o entendimento dos processos espaciais em outras áreas do conhecimento, como a Economia. O presente trabalho igualmente oferece evidências de que as diferenças econômicas possuem agudo componente geográfico.

\section{REFERÊNCIAS}

ABReU, J. F.; BARROSO, L.C.. Geografia, Modelo de Análise Espacial e GIS. Belo Horizonte: PUC Minas, 2003.

ABREU, J.F.; PAIVA, J.E. Sustentability and Human Development Index. In: SECONDINI, P. La Qualitá dello Spazio. Bologna: CLUEB, 2009.

ABREU, J. F.; MUZZARELLI, A. Introduzione ai Sistemi Informativi Geografici. Franco Angeli, Forum per la Tecnologia della Informazione. Università di Bologna e Pontifícia Universidade Católica de Minas Gerais. Milano, Italy, 2003.

AFFONSO, R. B. A. O federalismo e as teorias hegemônicas da economia do setor público na segunda metade do século XX: um balanço crítico. 2003. $281 \mathrm{f}$. Tese (Doutorado) - Instituto de Economia da Universidade Estadual de Campinas, Campinas, 2003.

AFONSO, J. R.; VILLELA, R. Evolução da carga tributária. Boletim Conjuntural (Nota Técnica), Rio de Janeiro, n. 28, jul., 1994.

AFONSO, J. R. R.; ARAUJO, E. A.; NÓBREGA, M. A. R. IPTU no Brasil, um diagnóstico abrangente. Rio de Janeiro: FGV Projetos/ Instituto Brasiliense de Direito Público (IDP), 2013.

ALMEIDA, E. Curso de Econometria Espacial Aplicada. Piracicaba: ESALQ-USP, 2004.

ALMEIDA, E. Econometria Espacial Aplicada. Campinas: Editora Alínea, 2012.

ANSELIN, L. Spatial Econometrics. In: BALTAGI, B. H. (Ed.). A companion to theoretical econometrics. Malden, MA: Blackwell Publishing Ltd., 2003.

ANSELIN, L. Interactive techniques and exploratory spatial data analysis. In: LONGLEY, P. A, GOODCHILD, M. F., MAGUIRE, D. J. \& RHIND, D. W. Geographical Information Systems. Volume 1. Principles and Technical Issues. New York: John Wiley \& Sons, Inc., 1999.

BENNETT, R. J. The Geography of Public Finance: welfare under fiscal federalism and local government finance. London/New York: Methuen, 1980. 
BERRY, B. Foreword. In.: SHEKAR, S. ; XIONG, H. (Ed.). Encyclopedia of GIS. New York: Springer Science Business Media, 2008.

BIRD, R. M. Fiscal Flows, Fiscal Balance, and Fiscal Sustainability. In: BIRD, R. M. ; VAILLANCOURT, F. (Ed.). Perspectives on fiscal federalism. Washington, DC: World Bank Institute, WBI Learning Resources Series, 2006.

BIVAND, R. Exploratory spatial data analysis. In: FISCHER, M. M. ; GETIS, A. (Ed.). Handbook of applied spatial analysis - software tools, methods and applications. Heidelberg Dordrech: Springer-Verlag, 2010.

BOADWAY, R.; SHAH, A. Overview, Fiscal Federalism - principles and practices of multiorder governance. Cambridge: Cambridge University Press, 2009.

BOWMAN, A.; KEARNEY, R. State and local government. Wadsworth: Cengage Learning, 2011.

BRASIL. Secretaria da Receita Federal. Carga tributária no Brasil - 2014 (análise por tributo e bases de incidência). Brasília: Ministério da Fazenda/Secretaria da Receita Federal, 2015a.

BRASIL. Secretaria do Tesouro Nacional. o que você precisa saber sobre as transferências fiscais da união - princípios básicos. Brasília: Ministério da Fazenda, 2015b.

BRASIL. Secretaria do Tesouro Nacional. Transferências Intergovernamentais. Brasília: Ministério da Fazenda/SURIN - Subsecretaria de Relações Financeiras Intergovernamentais para com Estados e Municípios/COINT - Coordenação-Geral de Análise e Informações das Transferências Financeiras Intergovernamentais, 2016. Disponível em: <http://www.esaf.fazenda.gov.br/assuntos/educacao-fiscal/pnef/ reunioes-de-trabalho/rio-de-janeiro/apresentacoes-1/transferenciasintergovernamentais-stn>. Acesso em: 17 jan. 2016.

BRENNAN, G.; BUCHANAN, J. M. The power to tax - analytical foundations of a fiscal constitution. Indianapolis: Liberty Fund, 2000a.

BURGESS, M. Federalism and political territoriality: the character and significance of constituent units in the new federal models. Rome, Italy. Paper prepared for the Annual Conference of the International Association of Centres for Federal Studies (IACFS), 19-21 September 2012 on the theme 'Federalism, Regionalism and Territory', 2012. Disponível em: <http://www.issirfa.cnr.it/download/ Michael\%20Burgess\%20\%28paper\%29.pdf>. Acesso em: 30 abr. 2016.

CLOKE, P. et al. Practising human geography. London: SAGE Publications, 2004.

COSSIO, F. A. B. Disparidades econômicas inter-regionais, capacidade de obtenção de recursos tributários, esforço fiscal e gasto público no federalismo brasileiro. 1998. 131 f. Dissertação (Mestrado) - Departamento de Economia da PUC/RJ, Rio de Janeiro, 1988.

DAHLBY, B. Opciones tributarias: sobre las competencias tributarias en un sistema federal. Revista Internacional de Ciências Sociales, $\mathrm{n}^{\circ}$ 167, 2001.

DIKSHIT, R. D. The political feography of federalism: an inquiry into origins and stability. Delhi: The Macmillan Company of India Limited, 1975.

ELAZAR, D. J. Anàlisi del federalisme. In: ELAZAR, D. J. Anàlisi del federalisme i altres textos. Generalitat de Catalunya: Institut d'Estudis Autonòmics (Clàssics del federalisme), 2011b.

ELAZAR, D. J. Federalism: an overview. Pretoria: HSRC Publishers, 1995. 
FOTHERINGHAM, A. S., BRUNSDON, C; CHARLTON, M. Geographically weighted regression - the analysis of spatially varying relationships. Chichester, West Sussex, England: John Wiley \& Sons Ltd., 2002.

FOTHERINGHAM, A. S. Quantification, Evidence and Positivism. In: AITKEN, S. ; VALENTINE, G. (Ed.). Approaches to human geography. London: Sage Publications Ltd., 2006.

FOTHERINGHAM, S.; BRUNSDON, C.; CHARLTON, M. Qualitative geography perspectives on spatial data analysis. London: Sage Publications Ltd., 2007.

GETIS, A.; ALDSTADT, J. Constructing the spatial weights matrix using a local statistic. In: ANSELIN, L. ; REY, S. J. (Ed.). Perspectives on spatial data analysis. Berlin/ Heidelberg: Springer, 2010.

GITELSON, A. R; DUDLEY, R. L.; DUBNICK, M. J. American government. Boston: Wadsworth, Cengage Learning, 2012.

GOODCHILD, M. F. Data Analysis, Spatial. In.: SHEKAR, S. ; XIONG, H. (Ed.). Encyclopedia of GIS. New York: Springer Science Business Media, 2008a.

GRODZINS, M. The federal system. In: WOLL, P. (Ed.). American government readings and cases. New York: Pearson Longman, 2004.

JOHNSTON, R. Sixty years of change in human geography. Paper prepared for the History of Postwar Social Science Seminars, London School of Economics, 25 April, 2006.

KITCHIN, R. Positivistic geographies and spatial science. In.: AITKEN, S. ; VALENTINE, G. (Ed.). Approaches to human geography. London: SAGE Publications, 2006.

LESAGE, J. P.; PACE, R. K. Introduction to spatial econometrics. Boca Raton, Florida: Taylor \& Francis Group, LLC, Statistics: a series of textbooks and monographs; 196, 2009.

LEUNG, YEE. Knowledge discovery in spatial data. Berlin Heidelberg: SpringerVerlag, 2010.

MARSHALL, A. A critique of the development of quantitative methodologies in human geography. 2011. Disponível em: <http://www.radstats.org.uk/no092/ marshall92.pdf>. Acesso em: 18 jul. 2014.

MCLURE, C. E. O problema da atribuição de impostos: considerações conceituais e de caráter administrativo para se alcançar a autonomia fiscal das unidades subnacionais. Stanford: Instituição Hoover, Universidade de Stanford, Curso de Relações Fiscais Intergovernamentais e Financeira de Governos Locais, organizado pelo Instituto de Desenvolvimento Econômico do Banco Mundial, Viena, Áustria, 1998. Disponível em: ^http://www.bndes.gov.br/SiteBNDES/export/sites/default/bndes_pt/Galerias/ Arquivos/bf_bancos/e0000747.pdf^. Acesso em: 16 maio 2016.

MENDES, M., MIRANDA, R. B.; COSIO, F. B. Transferências intergovernamentais no Brasil: diagnóstico e proposta de reforma. Brasília: Consultoria Legislativa do Senado Federal/Coordenação de Estudos, Textos para Discussão 40, 2008.

MORANDI, L.; E. J. REIS. Estoque de capital fixo no Brasil, 1950-2002. In: ENCONTRO NACIONAL DE ECONOMIA - ANPEC, 32, João Pessoa, PB, 2004 Anais ....João Pessoa. Disponível em: ^http://www.anpec.org.br/encontro2004/artigos/A04A042^. Acesso em: 30 jul. 2015.

MUSSON, S. The geography of the private finance initiative. Geographical Paper No. 188. University of Reading. Department of Geography. Geography: the University of Reading, 2009. 
MUSGRAVE, R.; MUSGRAVE, P. B. Finanças públicas; teoria e prática. Rio de Janeiro: Ed. Campus; São Paulo: Edit. Universidade de São Paulo, 1980.

MUSGRAVE, R. Teoria das finanças públicas. São Paulo: Editora Atlas, 1976.

MUWONGE, A.; EBEL, R. D. Finanzas intergubernamentales en un mundo descentralizado. In: FARVACQUE-VITKOVIC, C.; KOPANYI, M. (Ed.). Finanzas municipales: manual para los gobiernos locales. Washington, DC: Banco Mundial, 2015.

NAZARETH, P. A. C. P. Descentralização e federalismo fiscal: um estudo de caso dos Municípios do Rio de Janeiro. 2007. 311 f. Tese (Doutorado) - Instituto de Economia da Universidade Federal do Rio de Janeiro, Rio de Janeiro, 2007.

NELSON, T. A. Trends in spatial statistics, the professional geographer. 2011. Disponível em: <http://dx.doi.org/10.1080/00330124.2011.578540>. Acesso em: 30 abr. 2016.

OATES. W. E. An essay on fiscal federalism. In: BAIMBRIDGE, M. ; WHYMAN, P. (Ed.) Fiscal federalism and european economic integration. London: Routledge, Taylor \& Francis Group, 2004.

OATES. W. E. Fiscal federalism. New York: Jovanovich, 1972.

OLIVEIRA, F. A. Teorias da federação e do federalismo fiscal: o caso brasileiro. Belo Horizonte: Texto para Discussão n 43, Escola de Governo da Fundação João Pinheiro, 2007.

ORGANISATION FOR ECONOMIC CO-OPERATION AND DEVELOPMENT. Relatório Territorial da OCDE: Brasil 2013. Paris: OECD Publishing, 2013.

OYANA, T. J.; MARGAI, F. M. Spatial analysis - statistics, visualization, and computational methods. Boca Raton: CRC Press/Taylor \& Francis Group, 2016.

PEET, R. Modern geographical thought. Oxford: Blackwell, 1998.

PIANCASTELLI M.; BOUERI, R.; LIMA, E. P. Descentralização fiscal, harmonização tributária e relações intergovernamentais: como distintas federações reagem aos desafios da globalização. In: REZENDE, F. (Ed.). Desafios do federalismo fiscal. Rio de Janeiro: FGV, 2006.

REIS, G. A. dos. Federalismo e finanças municipais no Brasil: uma análise espacial. 415 f. Tese (Doutorado) - Pontifícia Universidade Católica de Minas Gerais. Programa de Pós-Graduação em Geografia. Belo Horizonte. 2016.

REZENDE, F.; AFONSO, J. R. The brazilian federation: facts, challenges, and perspectives. In: WALLACK, J. S. ; SRINIVASAN, T. N. Federalism and economic reform international perspectives. Cambridge: Cambridge University Press, 2006.

RODDEN, J. Federalism. In: WEINGAST, B. R. ; WITTMAN, D. A. (Ed.). The Oxford Handbook of political economy. Oxford: Oxford University Press, 2006.

ROSENN, K. S. Federalism in Brazil. Duquesne Law Review, v.43, p.577-598, 2005. RUBINSTEIN, F. Brazil. In: BIZIOLI, G. ; SACCHETTO, C. (Ed.). Tax aspects of fiscal federalism: a comparative analysis. Amsterdam: IBFD, 2011.

SANKEY, T. T. Statistical descriptions of spatial patterns. In.: SHEKAR, S., ; XIONG, H. (Eds.). Encyclopedia of GIS. New York: Springer Science Business Media, 2008.

SERRA, J.; R. AFONSO, J. R. R. El federalismo fiscal en Brasil: una visión panorâmica. Revista de la Cepal, v.91, p.27-52, abril, 2007. 
SHAH, A. A practitioner's guide to intergovernmental fiscal transfers. In: BOADWAY, $\mathrm{R}$. ; SHAH, A. (Ed.). Intergovernmental fiscal transfers: principles and practice. Washington: The World Bank, public sector governance and accountability series, 2007.

SHORT, J. R. An introduction to political geography. London: Routledge, 2003.

STILLWELL, J.; CLARKE, G. Introduction. In: STILLWELL, J. ; CLARKE, G. (Ed.) Applied GIS and spatial analysis. Chichester, West Sussex, England: John Wiley \& Sons Ltd., The Atrium, 2004.

TIEBOUT, C. M. A pure Theory of Local Expenditure. Journal of Political Economy, v.64, p.416-424, 1956.

VARGAS, N. C. A Descentralização e as teorias do federalismo fiscal. Ensaios FEE, Porto Alegre, v.32, n.1, p.51-76, jun, 2011.

VERGOLINO, J. R. O. Federalismo e autonomia fiscal dos governos estaduais no Brasil: notas sobre o período recente (1990-2010). In: MONTEIRO NETO, A. Governos estaduais no federalismo brasileiro: capacidades e limitações governativas em debate. Brasília: Ipea, 2014.

WAGNER, R. E. Design vs. emergence in a theory of federalism: toward institutional reconciliation. George Mason University, Department of Economics, Working Paper No. 15-24 (Draft paper for the annual meeting of the Public Choice Society, San Antonio, March 13-15, 2015.). 2015. Disponível em: <http://papers.ssrn.com/sol3/ cf_dev/AbsByAuth.cfm?per_id=238919>. Acesso em: 25 fev. 2016.

WANG, F. Quantitative methods and socio-economic applications in GIS. Boca Raton: CRC - Taylor \& Francis Group, 2015.

WATTS, R. L. Comparing Federal Systems in the 1990's. Kingston: Institute of Intergovernmental Relations, Queen's University, 1996.

Recebido em julho de 2018

Aceito em outubro de 2018 